\title{
VARIAÇÃO RADIAL DA DENSIDADE BÁSICA E DIMENSÕES CELULARES DA MADEIRA DE Cariniana legalis (Mart.) O. Kuntze EM FUNÇÃO DA PROCEDÊNCIA
}

\author{
Israel Luiz de Lima ${ }^{1}$, Eduardo Luiz Longui ${ }^{2}$, Michelle Fonseca Garcia ${ }^{3}$, Antonio Carlos Scatena Zanatto ${ }^{4}$, \\ Miguel Luiz Menezes Freitas ${ }^{5}$, Sandra Monteiro Borges Florsheim ${ }^{6}$
}

(recebido: 26 de julho de 2010; aceito: 30 de junho de 2011)

\begin{abstract}
RESUMO: Testes de procedência podem fornecer informações sobre o comportamento silvicultural e qualidade da madeira para a exploração da variabilidade e a conservação de material genético para usos futuros. Neste trabalho, objetivou-se estudar a variação radial da densidade básica e dimensões celulares da madeira de procedências de Cariniana legalis plantadas em Luiz Antonio SP. Mudas de três procedências (Porto Ferreira, Piracicaba e Campinas) foram plantadas em Luiz Antonio-SP, utilizando-se seis repetições em delineamento experimental de blocos casualizados. Após 26 anos do plantio, abateram-se dezoito árvores, seis de cada procedência. Foram estudadas a densidade básica e dimensões celulares da madeira. De acordo com os resultados, pode-se concluir que densidade básica, comprimento das fibras, espessura da parede das fibras, comprimento dos elementos de vaso, diâmetro de vasos, altura e largura de raios unisseriados foram influenciados pela procedência. Foi observada uma relação positiva entre comprimento das fibras, espessura da parede das fibras, comprimentos dos vasos, diâmetro dos vasos e altura de raios multisseriados com a posição radial e uma relação negativa entre a frequência de vasos com a posição radial.
\end{abstract}

Palavras-chave: Elementos anatômicos, anatomia da madeira, qualidade da madeira.

\section{RADIAL VARIATION OF BASIC DENSITY AND WOOD CELLS DIMENSIONS OF Cariniana legalis (Mart.) O. Kuntze DEPENDING ON THE PROVENANCE}

\begin{abstract}
Provenance tests can provide information about the silvicultural behavior and wood quality for the exploration of variability and conservation of genetic material for future use. This study aims to investigate the effect of provenances on some wood properties of the Cariniana legalis. Seedlings of three provenances (Porto Ferreira, Piracicaba and Campinas) were planted in Luiz Antonio-SP using randomized block design with six replicates. After 26 years of planting, eighteen trees, six of each provenance, were felled. The properties studied were basic density and the cellular dimensions. The results revealed that the basic density, fiber length, fiber wall thickness, vessel element length, vessel diameter, uniseriate ray height and width were influenced by the provenances. A good positive relationship was found among the fiber length, fiber wall thickness, vessel element length; vessel diameter and multiseriate ray height with radial position and a negative relation between vessel frequency with the radial position.
\end{abstract}

Key words: Anatomical elements, anatomy of the wood, quality of wood.

\section{INTRODUÇÃO}

O Instituto Florestal do Estado de São Paulo vem promovendo a conservação ex situ de várias espécies florestais nativas ameaçadas e com potencial econômico, desde a década de 1960, sendo pioneiro nessa área (LIMA et al., 2010). O Instituto possui experimentos na forma de testes de procedências, testes de progênies, testes combinados com procedências x progênies, populações base e pomares de sementes com mudas de diversas espécies. Esses plantios experimentais implantados e mantidos pelo Instituto Florestal, além de fornecer informações sobre o comportamento silvicultural das espécies, permitem a estimativa de parâmetros genéticos de características de interesse econômico, seleção de material genético, exploração da variabilidade e conservação da base genética para usos futuros (SIQUEIRA et al., 2000).

Entre as espécies pesquisadas pelo Instituto Florestal, destaca-se Cariniana legalis (Mart.) O. Kuntze, da família Lecythidaceae, é encontrada no

\footnotetext{
${ }^{1}$ Engenheiro Florestal, Pesquisador - Instituto Florestal do Estado de São Paulo - Divisão de Dasonomia - Rua do Horto, 931 - 02377-000 - São Paulo, SP - israelluizde.lima@yahoo.com.br

${ }^{2}$ Biólogo, Pesquisador - Instituto Florestal do Estado de São Paulo - Divisão de Dasonomia - Rua do Horto, 931 - 02377-000 - São Paulo, SP elongui@if.sp.gov.br

${ }^{3}$ Bióloga, Pesquisadora - Instituto Florestal do Estado de São Paulo - Rua do Horto, 931 - 02377-000 - São Paulo, SP - littleberry_mi@hotmail.com ${ }^{4}$ Engenheiro Agrônomo, Pesquisador - Instituto Florestal do Estado de São Paulo - Secretaria do Meio Ambiente - Estação Experimental de Luiz Antonio - 14210-000 - Luis Antônio, SP - acszanatto@if.sp.gov.br

${ }^{5}$ Engenheiro Agrônomo, Pesquisador Dr. em Genética e Melhoramento de Plantas - Instituto Florestal de São Paulo-Secretaria Meio Ambiente do Estado de São Paulo - Divisão de Dasonomia -Seção de Melhoramento Florestal - Rua do Horto, 931 -02377-000 - São Paulo, SP-miguellmfreitas@yahoo.com.br ${ }^{6}$ Bióloga, Pesquisadora - Instituto Florestal do Estado de São Paulo - Divisão de Dasonomia - Rua do Horto, 931 - 02377-000 - São Paulo, SP sflorsheim@if.sp.gov.br
}

Cerne, Lavras, v. 17, n. 4, p. 517-524, out./dez. 2011 
estrato superior da Floresta Ombrófila Densa (Floresta Atlântica), na formação baixo-montana e na Floresta Estacional Semidecidual (LORENZI, 2002), distribuindose, naturalmente, nos Estados do Espírito Santo, Rio de Janeiro, São Paulo, Minas Gerais, Mato Grosso, Bahia e Pernambuco (CARVALHO, 2003). É conhecida popularmente por diversos nomes, dependendo da região de ocorrência: caixão, coatinga, congolo-de-porco, cavinho-branco, estopa, jequitibá, jequitibá-agulheiro, jequitibá-branco, jequitibá-cedro, jequitibá-grande, jequitibá-rei, jequitibá-vermelho, jequitibá-rosa, paucaixão, pau-de-cerne, sapucaia-de-apito, pau-carga e sapucaia-de-assovio (CARVALHO, 2003).

Segundo Mainieri e Chimelo (1989), C. legalis pode atingir a altura de $30-50 \mathrm{~m}$, com tronco de $70-100$ $\mathrm{cm}$ de DAP (diâmetro à altura do peito, ou seja, a $1,30 \mathrm{~m}$ do solo), apresenta folhas membranáceas, glabras, de 4-7 $\mathrm{cm}$ de comprimento por 2-4 $\mathrm{cm}$ de largura, flores pequenas da cor creme e fruto pixídio lenhoso. Trata-se de uma das maiores árvores da flora brasileira e da Região Sudeste (CARVALHO, 2003; LORENZI, 2002). O crescimento da espécie varia de moderado a rápido. Em alguns plantios, $o$ crescimento superou $21 \mathrm{~m}^{3}$. ha ${ }^{-1}$. ano ${ }^{-1}$ (CARVALHO, 2003).

A madeira de $C$. legalis apresenta cerne geralmente róseo-acastanhado ou bege-rosado, ou ainda bege-rosaescuro, eventualmente com sombras pardacentas, alburno pouco diferenciado, bege-claro; grã-direta; textura média, uniforme, superfície áspera ao tato, cheiro e gosto imperceptíveis, macia ao corte, com densidade aparente de cerca de $530 \mathrm{~kg} \mathrm{~m}^{-3}$. Segundo observações práticas a respeito de sua utilização, a madeira de $C$. legalis, quando exposta a condições adversas é considerada de baixa resistência ao ataque de organismos xilófagos (MAINIERI; CHIMELO, 1989). Utilizada para a construção civil, papel, bem como obras internas, para contraplacados, folhas faqueadas, móveis, para confecção de brinquedos, salto de calçados, lápis, cabos de vassoura (CARVALHO, 2003; LORENZI, 2002). Também produz celulose para papel de boa qualidade, com um teor de lignina de $24,2 \%$. As fibras da pasta celulósica apresentam um comprimento médio de $1,35 \mathrm{~mm}$ e largura média de 0,02 mm, conforme Carvalho (2003).

Neste trabalho, objetivou-se estudar a variação radial da densidade básica e dimensões celulares da madeira de três procedências de $C$. legalis plantadas em Luiz Antonio, SP.

\section{MATERIAL E MÉTODOS}

Neste trabalho foram utilizadas amostras de madeira de árvores plantadas na Estação Experimental de Luiz Antonio-SP (EELA), do Instituto Florestal. As árvores são oriundas de sementes de polinização abertas colhidas em três populações de C. legalis, (Porto Ferreira, Piracicaba e Campinas, Estado de São Paulo). O ensaio foi implantado em 1982, na EELA, localizada nas coordenadas $21^{\circ} 40^{\prime} \mathrm{S}, 47^{\circ} 49^{\prime} \mathrm{W}$, altitude de $550 \mathrm{~m}$, possuindo clima tropical (Cwa), inverno seco, precipitação média anual de $1.280 \mathrm{~mm}$ e sendo o solo do tipo Latossolo Vermelho (SEBBENN et al., 2000). O delineamento experimental adotado foi o de blocos de famílias compactas (WRIGHT, 1978), contendo três procedências e seis repetições. O espaçamento utilizado foi o de 3,0 x 3,0 m. Coletaram-se amostras de madeira de 18 árvores aos 26 anos para o ensaio tecnológico, sendo três procedências, selecionadas dentro de cada um dos seis blocos, no talhão, tiradas da classe de DAP médio das parcelas, o qual foi definido por um inventário florestal exploratório. De cada árvore, foi retirado um disco de $7 \mathrm{~cm}$ de espessura na região do DAP. De cada disco retiraram-se amostras no sentido medulacasca, correspondendo ao raio da árvore (Figura 1).

A partir das amostras, foram preparados corpos de prova, de dimensões de aproximadamente $(2 \times 2 \times$ $2 \mathrm{~cm}$ ) de aresta, para o estudo da variação radial do lenho das características: densidade básica (DB), comprimento das fibras (CF), espessura da parede das fibras (EPF), comprimentos dos elementos de vaso $(\mathrm{CV})$, diâmetro dos vasos (DV), frequência dos vasos (FV), frequência dos raios (FR), altura dos raios multisseriados (ARM), altura dos raios unisseriados (ARU), largura dos raios multisseriados (LRM), largura dos raios unisseriados (LRU). Para a obtenção de secções histológicas, os corpos de prova $\left(2 \mathrm{~cm}^{3}\right)$ foram amolecidos por meio de cozimento em água e glicerina (4:1), até apresentarem condições ideais ao corte. Seç̧ões entre 20 e $30 \mathrm{~mm}$ nos planos transversal e longitudinal tangencial foram obtidas com auxílio de micrótomo de deslize marca Leitz 1208. As secções foram clarificadas com hipoclorito de sódio (60\%), lavadas em água, coradas com safranina 1\% (JOHANSEN, 1940) e montadas em solução de água com glicerina. O lenho dissociado foi preparado de acordo com o método de Franklin modificado (BERLYN; MIKSCHE, 1976), corado com safranina alcoólica e montado em solução de água com glicerina (1:1). A terminologia empregada para as análises anatômicas seguiu as recomendações do IAWA Committee (1989). Todas as mensurações foram realizadas em microscópio óptico trinocular, acoplado a uma câmera de vídeo para captura de imagens e sistema de medições (Marca Olympus modelo BX 50 com software de análise de imagens Image - Pro Express versão 6.3).

Cerne, Lavras, v. 17, n. 4, p. 517-524, out./dez. 2011 


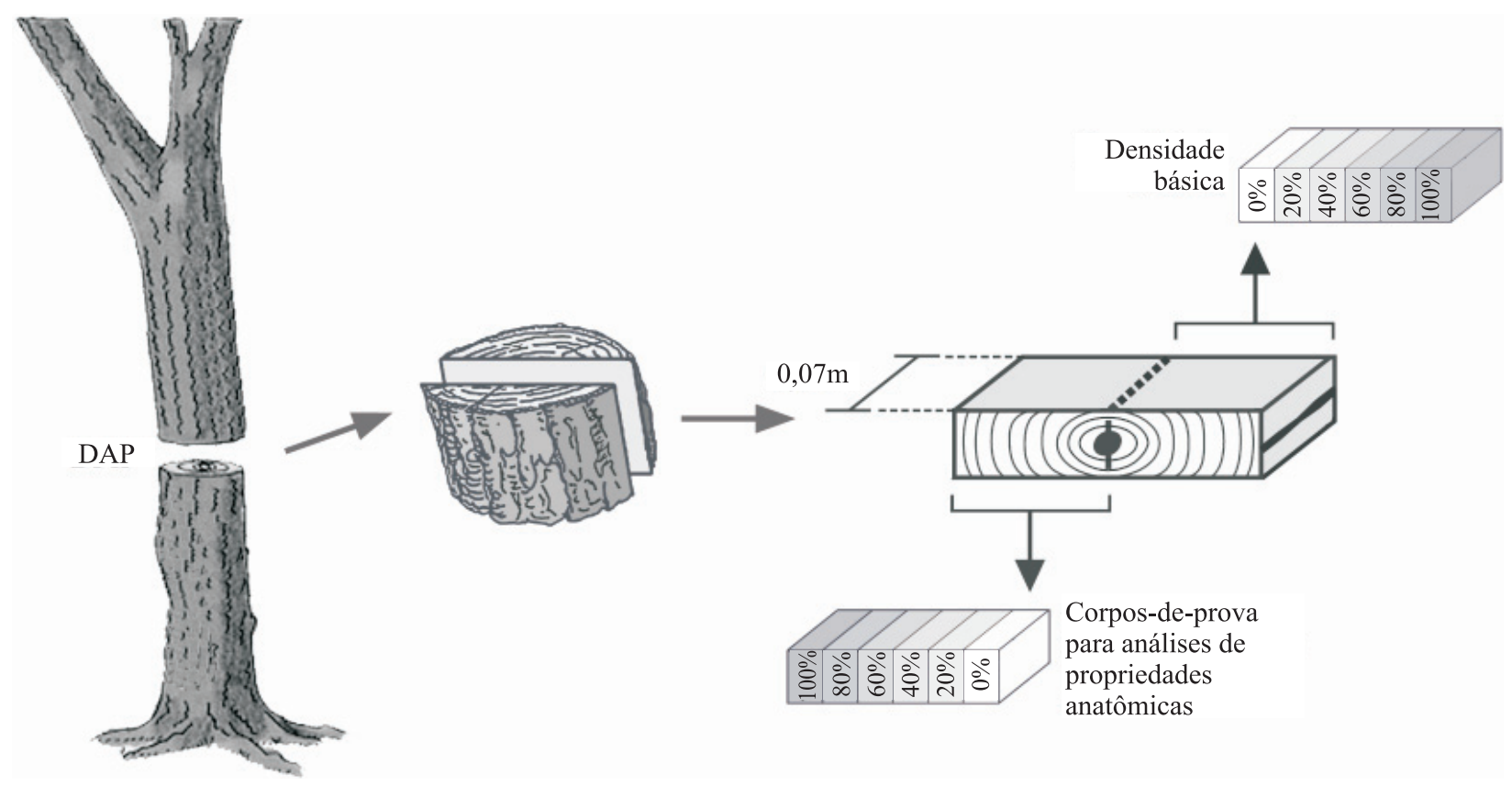

Figura 1 - Posição de retirada dos discos nas árvores e corpos de prova para a caracterização da madeira de Cariniana legalis.

Figure 1 - Sampling position of the discs in the trees and specimen for wood characterization of Cariniana legalis.

Para amostrar todas as variáveis foram adotadas $n=25$ medições. Na obtenção da análise da densidade básica, foi utilizado o método do máximo teor de umidade, segundo Foelkel et al. (1971).

As hipóteses testadas foram: a) a densidade básica e dimensões celulares da madeira, de diferentes procedências de $C$. legalis avaliadas aos 26 anos não diferem entre si; b) Não existem variações na densidade básica e dimensões celulares ao longo do raio das toras de C. legalis.

Na avaliação do experimento foi efetuado o teste de homogeneidade de variância por meio do teste de Hartley e, posteriormente, foi feito o teste $\mathrm{F}$ de análise de variância segundo o delineamento experimental de blocos casualizados. Foi aplicado o teste de Tukey, para verificarem-se diferenças significativas entre as médias sempre que o teste $\mathrm{F}$ detectou pelo menos uma diferença entre tratamentos ao nível de 5\% de significância. Estudouse também a relação entre as dimensões das fibras, densidade básica e a posição radial e, para isso, utilizou-se análise de regressão, ao nível de probabilidade de $95 \%$. $\mathrm{Na}$ análise dos dados, foi utilizado o procedimento PROC GLM e PROC REG do programa estatístico SAS (SAS INSTITUTE, 1999).

\section{RESULTADOS E DISCUSSÃO}

O resumo da análise de variância das propriedades em estudo está apresentado na Tabela 1. Entre as procedências foram observadas diferenças na densidade básica, comprimento de fibras, espessura da parede da fibra, comprimento de elementos de vaso, altura e largura dos raios unisseriados. Porém, não ocorreram diferenças entre procedências para diâmetro de vaso, frequência de vaso, altura e largura dos raios multisseriados.

A densidade básica da madeira de $C$. legalis variou significativamente entre procedências, porém, somente Piracicaba e Campinas diferiram entre si, sendo que Piracicaba apresentou o maior valor e Campinas, o menor (Tabela 2). Em trabalho similar, Andrade et al. (2009) também observaram variações na densidade básica entre três diferentes procedências de Gallesia integrifolia.

No presente estudo, o comprimento e a espessura da parede da fibra variaram significativamente entre procedências, sendo que o comprimento das fibras observado em Piracicaba diferiu de Campinas e a espessura da parede das fibras em Porto Ferreira diferiu significativamente de Piracicaba e Campinas (Tabela 2). O comprimento de elemento de vaso de Porto Ferreira diferiu significativamente de Piracicaba e Campinas (Tabela 2).

Cerne, Lavras, v. 17, n. 4, p. 517-524, out./dez. 2011 
Tabela 1 - Resumo da análise de variância efetuada para densidade básica (DB), comprimento de fibra (CF), espessura da parede da fibra (EPF), comprimento de elementos de vaso (CV), diâmetro de vaso (DV), frequência de vasos (FV), frequência de raio (FR), altura dos raios multisseriados (ARM), altura dos raios unisseriados (ARU), largura dos raios multisseriados (LRM), largura dos raios unisseriados (LRU) de Cariniana legalis aos 26 anos de idade.

Table 1 - Summary of analysis of variance of basic density (DB), fiber length (CF), fiber wall thickness (EPF), vessel element length $(C V)$, vessel diameter $(D V)$, vessel frequency $(F V)$, ray frequency $(F R)$, multiseriate ray height $(A R M)$, uniseriate ray height (ARU) multiseriate ray width (LRM) and uniseriate ray width (LRU) of Cariniana legalis 26-years-old.

\begin{tabular}{|c|c|c|c|c|c|c|c|c|c|c|c|c|}
\hline \multirow[b]{2}{*}{ Causa de variação } & \multirow[b]{2}{*}{ GL } & \multicolumn{11}{|c|}{ Quadrado médio } \\
\hline & & $\begin{array}{c}\text { DB } \\
\left(\mathrm{kg} \cdot \mathrm{m}^{-3}\right)\end{array}$ & $\begin{array}{c}\mathrm{CF} \\
(\mathrm{mm})\end{array}$ & $\begin{array}{l}\mathrm{EPF} \\
(\mu \mathrm{m})\end{array}$ & $\begin{array}{c}\mathrm{CV} \\
(\mu \mathrm{m})\end{array}$ & $\begin{array}{c}\text { DV } \\
(\mu \mathrm{m})\end{array}$ & $\begin{array}{c}\mathrm{FV} \\
\left(\mathrm{n}^{\mathrm{o}} \mathrm{mm}^{-2}\right)\end{array}$ & $\begin{array}{c}\mathrm{FR} \\
\left(\mathrm{n}^{\mathrm{o}} \mathrm{mm}^{-1}\right)\end{array}$ & $\begin{array}{l}\text { ARM } \\
(\mu \mathrm{m})\end{array}$ & $\begin{array}{l}\text { ARU } \\
(\mu \mathrm{m})\end{array}$ & $\begin{array}{l}\text { LRM } \\
(\mu \mathrm{m})\end{array}$ & $\begin{array}{l}\text { LRU } \\
(\mu \mathrm{m})\end{array}$ \\
\hline Bloco & 5 & 7017 & 0,1402 & 0,3296 & 3198 & 286 & 21,92 & 0,51 & 15594 & 4853 & 92,86 & 17,60 \\
\hline Procedência (P) & 2 & $3363^{*}$ & $0,0762^{*}$ & $0,9471^{* *}$ & $8748^{*}$ & $36,07^{\text {n.s. }}$ & $1,81^{\text {n.s. }}$ & $1,11^{\text {n.s. }}$ & $985^{\text {n.s. }}$ & $10233^{* *}$ & $0,12^{\text {n.s. }}$ & $76,30^{*}$ \\
\hline Posição radial (PR) & 6 & $3473^{*}$ & $0,2274^{* *}$ & $0,6667^{* *}$ & $34969^{* *}$ & $5792^{* *}$ & $207,67^{* *}$ & $1,01^{\text {n.s. }}$ & $15639^{* *}$ & $1155^{\text {n.s. }}$ & $17,38^{\text {n.s. }}$ & $26,30^{\text {n.s. }}$ \\
\hline$(\mathrm{P}) \times(\mathrm{PR})$ & 10 & $201^{\text {n.s. }}$ & $0,0089^{\text {n.s. }}$ & $0,0770^{\text {n.s. }}$ & $1052^{\text {n.s. }}$ & $174^{\text {n.s. }}$ & $6,57^{\text {n.s. }}$ & $0,32^{\text {n.s. }}$ & $2816^{\text {n.s. }}$ & $614^{\text {n.s. }}$ & $10,73^{\text {n.s. }}$ & $30,93^{\text {n.s. }}$ \\
\hline Resíduo & 85 & 1014 & 0,0214 & 0,0634 & 1352 & 101,79 & 7,32 & 0,53 & 2952 & 688 & 17,37 & 23,86 \\
\hline Média & & 488 & 1,61 & 3,34 & 467 & 109,76 & 15,98 & 6,66 & 363 & 199 & 33,09 & 18,55 \\
\hline $\mathrm{CV}_{\mathrm{e}}(\%)$ & & 6,52 & 9,09 & 7,53 & 7,85 & 9,19 & 16,93 & 10,93 & 14,96 & 13,14 & 12,59 & 26,32 \\
\hline
\end{tabular}

Onde: ** significativo ao nível de $1 \%$ de significância; $*$ significativo ao nível de $5 \%$ de significância, n. $\mathrm{s}=$ não significativo; $\mathrm{DP}=$ desvio padrão e $\mathrm{CVe}=$ coeficiente de variação experimental.

Tabela 2 - Médias da densidade básica (DB), comprimento das fibras (CF), espessura da parede das fibras (EPF), comprimentos dos elementos de vaso $(\mathrm{CV})$, diâmetro dos vasos (DV), frequência dos vasos (FV), frequência dos raios (FR), altura dos raios multisseriados (ARM), altura dos raios unisseriados (ARU), largura dos raios multisseriados (LRM), largura dos raios unisseriados (ARU) de Cariniana legalis aos 26 anos de idade.

Table 2 - Average basic density (DB), fiber length (CF), fiber wall thickness (EPF), vessel element length (CV); vessel diameter $(D V)$, vessel frequency $(F V)$, ray frequency $(F R)$, multiseriate ray height (ARM), uniseriate ray height (ARU), multiseriate ray width (LRM) and uniseriate ray width (ARU) of Cariniana legalis 26-years-old

\begin{tabular}{|c|c|c|c|c|c|c|c|c|c|c|c|}
\hline Tratamento & $\begin{array}{c}\text { DB } \\
\left(\mathrm{kg} \cdot \mathrm{m}^{-3}\right)\end{array}$ & $\begin{array}{c}\mathrm{CF} \\
(\mathrm{mm})\end{array}$ & $\begin{array}{l}\text { EPF } \\
(\mu \mathrm{m})\end{array}$ & $\begin{array}{c}\text { CV } \\
(\mu \mathrm{m})\end{array}$ & $\begin{array}{c}\text { DV } \\
(\mu \mathrm{m})\end{array}$ & $\begin{array}{c}\mathrm{FV} \\
\left(\mathrm{n}^{\mathrm{o}} \mathrm{mm}^{-2}\right)\end{array}$ & $\begin{array}{c}\mathrm{FR} \\
\left(\mathrm{n}^{\mathrm{o}} \mathrm{mm}^{-1}\right)\end{array}$ & $\begin{array}{l}\text { ARM } \\
(\mu \mathrm{m})\end{array}$ & $\begin{array}{l}\text { ARU } \\
(\mu \mathrm{m})\end{array}$ & $\begin{array}{l}\text { LRM } \\
(\mu \mathrm{m})\end{array}$ & $\begin{array}{l}\text { LRU } \\
(\mu \mathrm{m})\end{array}$ \\
\hline \multicolumn{12}{|l|}{ Procedência } \\
\hline Porto Ferreira & $485^{\mathrm{ab}}$ & $1,60^{\mathrm{ab}}$ & $3,52^{\mathrm{a}}$ & $485^{\mathrm{a}}$ & $109^{\mathrm{a}}$ & $16,23^{\mathrm{a}}$ & $6,46^{\mathrm{a}}$ & $365^{\mathrm{a}}$ & $218^{\mathrm{a}}$ & $33,10^{\mathrm{a}}$ & $20,16^{\mathrm{a}}$ \\
\hline Piracicaba & $499^{a}$ & $1,65^{\mathrm{a}}$ & $3,29^{\mathrm{b}}$ & $461^{\mathrm{b}}$ & $108^{\mathrm{a}}$ & $15,89^{\mathrm{a}}$ & $6,80^{\mathrm{a}}$ & $357^{\mathrm{a}}$ & $187^{\mathrm{b}}$ & $33,02^{\mathrm{a}}$ & $17,32^{\mathrm{b}}$ \\
\hline Campinas & $480^{\mathrm{b}}$ & $1,56^{\mathrm{b}}$ & $3,20^{\mathrm{b}}$ & $456^{\mathrm{b}}$ & $110^{\mathrm{a}}$ & $15,81^{\mathrm{a}}$ & $6,70^{\mathrm{a}}$ & $366^{\mathrm{a}}$ & $192^{\mathrm{b}}$ & $33,14^{\mathrm{a}}$ & $18,18^{\mathrm{ab}}$ \\
\hline \multicolumn{12}{|l|}{ Variação radial } \\
\hline Posição radial $(0 \%)$ & $499^{\mathrm{a}}$ & $1,40^{\mathrm{b}}$ & $3,06^{b}$ & $397^{\mathrm{d}}$ & $84^{\mathrm{e}}$ & $21,48^{\mathrm{a}}$ & $6,75^{\mathrm{a}}$ & $336^{\mathrm{bc}}$ & $202^{\mathrm{a}}$ & $33,73^{\mathrm{a}}$ & $18,96^{\mathrm{a}}$ \\
\hline Posição radial (20\%) & $493^{\mathrm{a}}$ & $1,57^{\mathrm{a}}$ & $3,28^{\mathrm{b}}$ & $453^{c}$ & $95^{\mathrm{d}}$ & $18,46^{\mathrm{b}}$ & $6,81^{\mathrm{a}}$ & $322^{c}$ & $187^{\mathrm{a}}$ & $31,25^{\mathrm{a}}$ & $17,07^{\mathrm{a}}$ \\
\hline Posição radial (40\%) & $488^{\mathrm{ab}}$ & $1,62^{\mathrm{a}}$ & $3,28^{b}$ & $457^{\mathrm{c}}$ & $107^{\mathrm{c}}$ & $15,70^{\mathrm{c}}$ & $6,96^{\mathrm{a}}$ & $355^{\mathrm{abc}}$ & $202^{\mathrm{a}}$ & $33,42^{\mathrm{a}}$ & $17,61^{\mathrm{a}}$ \\
\hline Posição radial (60\%) & $494^{\mathrm{a}}$ & $1,63^{\mathrm{a}}$ & $3,29^{b}$ & $473^{\mathrm{bc}}$ & $115^{\mathrm{bc}}$ & $14,35^{\mathrm{cd}}$ & $6,68^{\mathrm{a}}$ & $383^{\mathrm{ab}}$ & $195^{\mathrm{a}}$ & $33,62^{\mathrm{a}}$ & $18,35^{\mathrm{a}}$ \\
\hline Posição radial (80\%) & $493^{\mathrm{a}}$ & $1,69^{\mathrm{a}}$ & $3,57^{\mathrm{a}}$ & $497^{\mathrm{ab}}$ & $124^{\mathrm{ab}}$ & $13,20^{\mathrm{cd}}$ & $6,41^{\mathrm{a}}$ & $394^{\mathrm{a}}$ & $197^{\mathrm{a}}$ & $33,72^{\mathrm{a}}$ & $20,54^{\mathrm{a}}$ \\
\hline Posição radial (100\%) & $460^{\mathrm{b}}$ & $1,71^{\mathrm{a}}$ & $3,55^{\mathrm{a}}$ & $527^{\mathrm{a}}$ & $131^{\mathrm{a}}$ & $12,68^{\mathrm{d}}$ & $6,34^{\mathrm{a}}$ & $385^{\mathrm{ab}}$ & $211^{\mathrm{a}}$ & $33,80^{\mathrm{a}}$ & $18,78^{\mathrm{a}}$ \\
\hline
\end{tabular}

Obs: Médias seguidas de letras diferentes em uma mesma coluna diferem entre si (ao nível de 5\% de significância).

A largura dos raios unisseriados de Porto Ferreira diferiu de Piracicaba (Tabela 2). De acordo com Andrade et al. (2009), essas variações encontradas nas características analisadas, podem ter relação com as coordenadas geográficas, altitude e características ambientais dos locais de origem da coleta das sementes (procedências), ao receberem informações genéticas das árvores-mãe, mas em consequência da nova situação ambiental (local de plantio do ensaio), as árvores expressaram variações fenotípicas influenciadas pelo ambiente.

Cerne, Lavras, v. 17, n. 4, p. 517-524, out./dez. 2011 
Para o fator posição radial apenas a frequência, altura e largura dos raios unisseriados e multisseriados não diferiram entre si (Tabela 1). Também pode-se verificar que não ocorreu interação significativa, entre procedências e posição radial demonstrando não existir dependência entre esses fatores analisados (Tabela 1).

Na variação radial da densidade básica, as posições diferiram significativamente entre si. A posição radial $100 \%$ (próxima da casca) apresentou a menor densidade básica e a posição $0 \%$ (próxima da medula) a maior (Tabela 2). Pode-se observar também que ocorreu uma tendência de diminuição da densidade no sentido medulacasca. Resultado semelhante foi observado por Yanchuk e Micko (1990), porém, diferentemente da maioria dos trabalhos que estudaram a variação no sentido medulacasca para densidade básica dos gêneros Eucalyptus

(a)

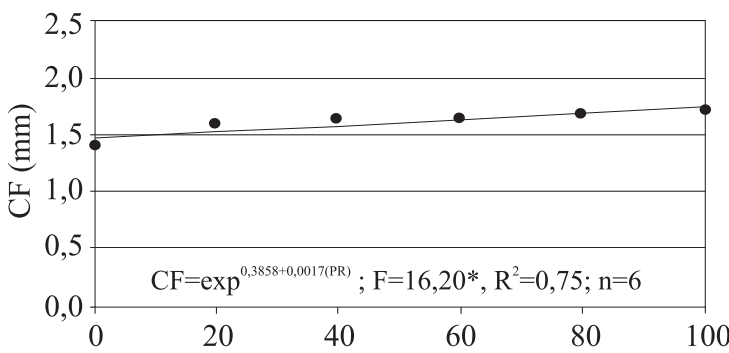

(c)

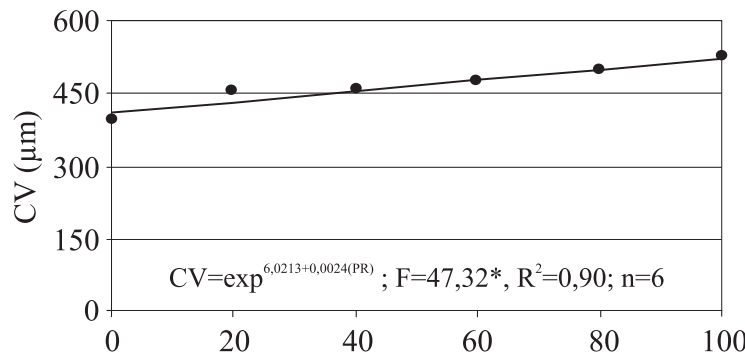

(e)

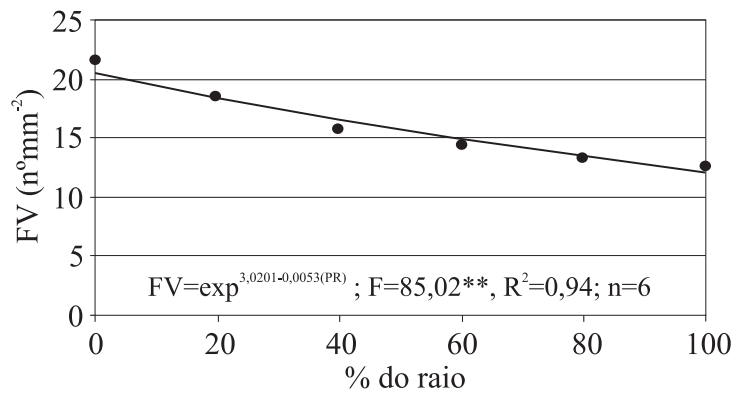

e Pinus, nos quais os autores relataram aumento na densidade no mesmo sentido (MALAN, 1991; MALAN; HOON, 1992; POLLI et al., 2006; WILKES, 1984, 1990). Essa tendência observada pode ser uma característica dessa espécie.

O comprimento e a espessura da parede das fibras apresentaram uma tendência de aumentar no sentido medula-casca (Figura 2). Resultado semelhante foi observado por Butterfield et al. (1993), Rocha et al. (2004) e Yanchuk e Micko (1990). Essa tendência de aumento do comprimento de fibra e uma estabilização a partir da posição $80 \%$ pode-ser um indicio de mudança de região de madeira juvenil para adulta (MALAN, 1995). De acordo com Wilkes (1988), o desenvolvimento de células com maiores dimensões, conforme a árvore fica mais velha pode estar relacionado com a maturação do lenho.

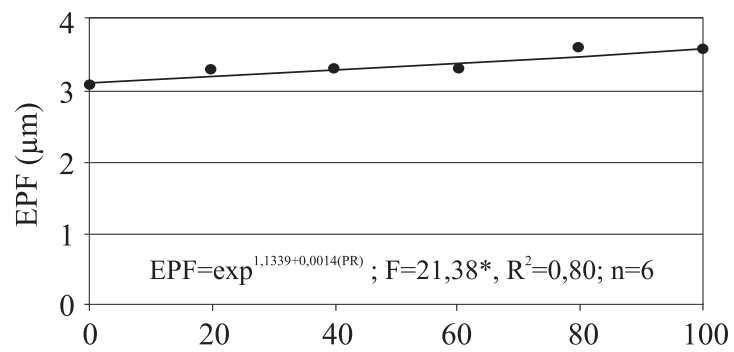

(b)

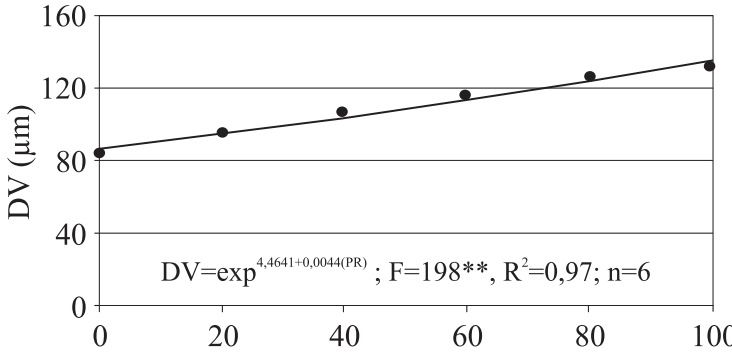

(d)

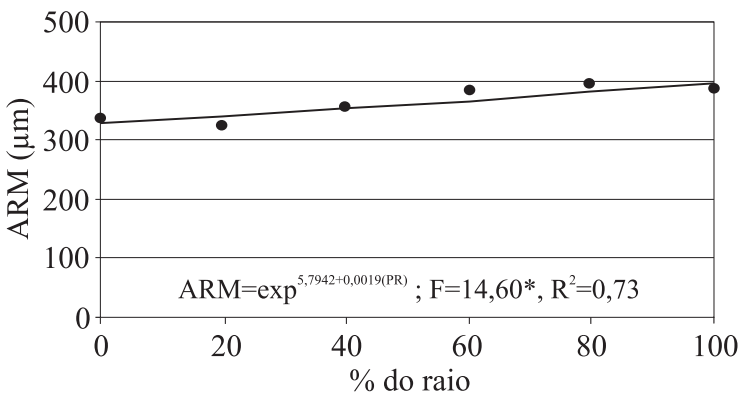

Figura 2 - Relações entre posição radial (PR) e comprimento das fibras (CF) [a], espessura da parede das fibras (EPF) [b], comprimento dos elementos de vaso (CV) [c], diâmetro dos vasos (DV) [d], frequência de vasos (FV) [e] e altura de raio multisseriados (ARM) [F] para Cariniana legalis, aos 26 anos de idade.

Figure 2 - Relations among the radial positions (PR) and fiber length (CF), [a], fiber wall thickness (EPF) [b], vessel element length $(C V)[c]$; vessel diameter $(D V)[d]$, vessel frequency $(F V)[e]$ and multiseriate ray height (ARM) [f] of Cariniana legalis of 26-years-old.

Cerne, Lavras, v. 17, n. 4, p. 517-524, out./dez. 2011 
Nas dimensões dos elementos de vasos ocorreram diferenças entre as posições radiais, porém enquanto o comprimento e diâmetro de vaso tendem a aumentar no sentido medula-casca, a frequência tende a diminuir (Tabela 2). Esse mesmo padrão foi observado por Florsheim et al. (2009) para populações de Eucalyptus dunnii e Ishiguri et al. (2009) para Paraserianthes falcataria. $\mathrm{O}$ aumento no diâmetro dos vasos na direção as casca também foi reportado em espécies nativas, como nos estudos de Longui et al. (2009) com Luehea divaricata e Dünisch et al. (2004) com Ilex paraguariensis.

$\mathrm{Na}$ Figura 3, verifica-se o aumento do diâmetro de vasos no sentido medula-casca, sendo que a posição medula diferiu significativamente da posição casca. Com relação às dimensões dos raios, apenas a altura dos raios multisseriados variou significativamente entre posições radiais que estão de acordo com Urbinati et al. (2003).

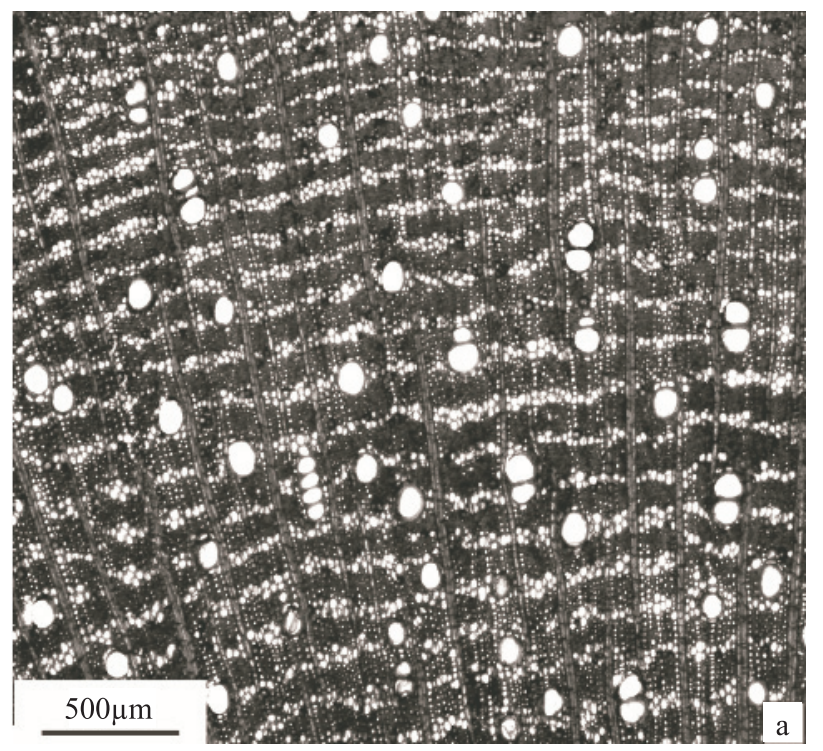

Para explicar melhor as variações radiais, foi realizado um estudo de relações entre as dimensões celulares e as posições radiais. Na Figura 2, temos os modelos de regressões que foram significativos, ou seja, que melhor se ajustaram às variações. $\mathrm{O}$ melhor modelo ajustado foi o logarítmico. De acordo com esse modelo de regressão, verifica-se que ocorreu uma tendência de aumento no sentido medula-casca para: comprimento das fibras, espessura da parede das fibras, comprimento dos elementos de vaso, diâmetro dos vasos e altura de raios multisseriados, já, para a frequência de elemento de vasos ocorreu uma tendência de diminuição. A densidade básica, frequência de raio, altura e largura de raios unisseriados e largura de raio multisseriados não apresentaram nenhum modelo significativo na variação medula-casca. Essas variações nas dimensões celulares são decorrentes do aumento gradativo da proporção de madeira adulta em relação à madeira juvenil, no sentido medula-casca (MALAN, 1995).

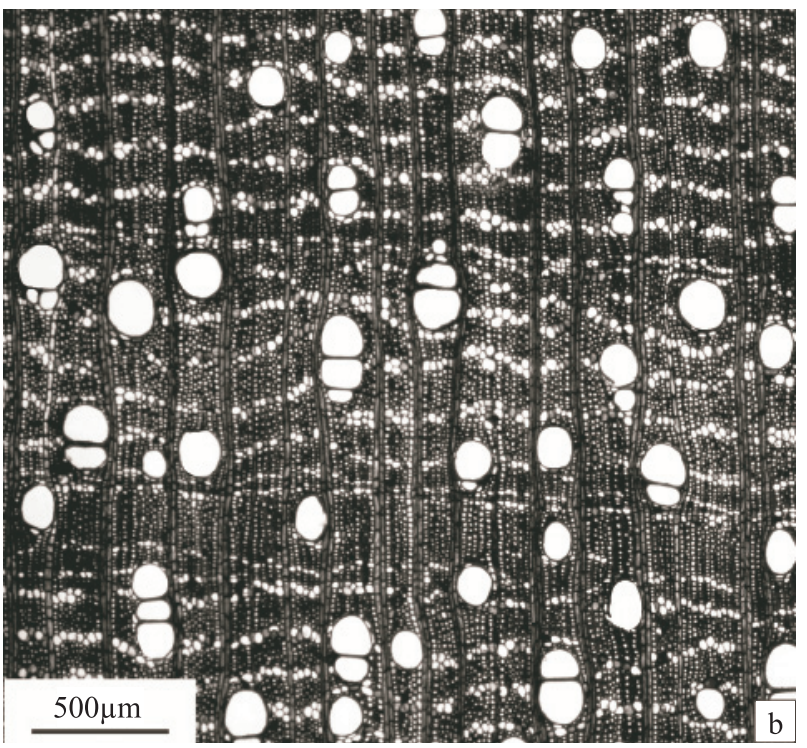

Figura 3 - Fotomicrografias do lenho de Cariniana legalis - a e b. Secções transversais da região da medula e da casca respectivamente. Notar aumento no diâmetro dos vasos (Figuras a e b). Barra $=500 \mu \mathrm{m}$.

Figure 3 - Photomicrographs of Cariniana legalis wood. - a and b. Transverse sections of the pith region and the bark region respectively. Please note the increase in vessel diameter (Figures a and b). Bar $=500 \mu \mathrm{m}$.

\section{CONCLUSÕES}

De acordo com os resultados obtidos para a densidade básica e características anatômicas avaliadas na madeira de Cariniana legalis de 26 anos, conclui-se que: - a densidade básica, comprimento de fibras, espessura da parede da fibra, comprimento de elementos de vaso, altura e largura dos raios unisseriados foram influenciados pela procedência;

- o diâmetro de vaso, frequência de vaso, altura e largura dos raios multisserirados não foram influenciados pela procedência;

- ocorreu uma tendência de aumento no sentido medula-casca para: comprimento das fibras, espessura da

Cerne, Lavras, v. 17, n. 4, p. 517-524, out./dez. 2011 
parede das fibras, comprimento dos elementos de vaso, diâmetro dos vasos e altura dos raios multisseriados.

- a frequência de elementos de vaso tendeu a diminuir no sentido da medula-casca;

- a densidade básica, frequência de raio, altura de raio unisseriados, largura de raio multisseriados e largura de raio unisseriados não apresentaram nenhuma tendência significativa de variação no sentido medula-casca.

\section{REFERÊNCIAS}

ANDRADE, I. M.; LONGUI, E. L.; LIMA, I. L.; FLORSHEIM, S. M. B. Efeito da procedência na variação radial do comprimento de fibras e densidade básica da madeira de Gallesia integrifolia (Spreng.) Harms. IF Série Registro, São Paulo, v. 40, p. 39-43, 2009.

BERLYN, G. P.; MIKSCHE, J. P. Botanical microtechnique and cytochemistry. Iowa: The Iowa University, 1976. 326 p.

BUTTERFIELD, R. P.; COOK, R. P.; ADAMS, R.; MORRIS, $\mathrm{R}$. Radial variation in wood specific gravity, fibre length and vessel area for two central american hardwoods: Hyeronima alchorneoides and Vochysia guatemalensis: natural and plantation-grown trees. IAWA Journal, Leiden, v. 14, n. 2, p. 153-161, 1993.

CARVALHO, P. E. R. Espécies arbóreas brasileiras. Brasília: Embrapa Informações Tecnológica; Colombo: Embrapa Floresta, 2003. 739 p.

DÜNISCH, O.; REISSMANN, C. B.; OLISZESKI, A. $\mathrm{V}$. Variability of vessel characteristics in xylem of Ilex paraguariensis (mate-tree) from south Brazil. IAWA Journal, Leiden, v. 25, n. 4, p. 449-458, 2004.

FLORSHEIM, S. M. B.; COUTO, H. T. Z.; LIMA, I. L.; LONGUI, E. L. Variação nas dimensões dos elementos anatômicos da madeira de Eucalyptus dunnii aos sete anos de idade. Revista do Instituto Florestal, São Paulo, v. 21, n. 1, p. 79-91, 2009.

FOELKEL, C. E. B.; BRASIL, M. A. M.; BARRICHELO, L. E. G. Métodos para determinação da densidade básica de cavacos para coníferas e folhosas. IPEF, Piracicaba, n. 2/3, p. 65-74, 1971.

IAWA COMMITEE. List microscope features of hardwood identification. IAWA Bulletin, Leiden, v. 10, n. 3, p. 221-259, 1989.
ISHIGURI, F.; HIRAIWA, T.; IIZUKA, K.; YOKOTA, S.; PRIADI, D.; SUMIASRI, N.; YOSHIZAWA, J. Radial variation of anatomical characteristics in Paraserianthes falcataria planted in Indonesia. IAWA Bulletin, Leiden, v. 30, n. 3, p. 343-352, 2009.

JOHANSEN, D. A. Plant microtecniques. New York: McGraw-Hill, 1940. 523 p.

LIMA, I. L.; LONGUI, E. L.; ANDRADE, I. M.; GARCIA, J. N.; ZANATTO, A. C. S.; MORAIS, E.; FLORSHEIM, S. M.

B. Efeito da procedência em algumas propriedades da madeira de Gallesia integrifolia (Spreng.) Harms. Revista do Instituto Florestal, São Paulo, v. 22, n. 1, p. 61-69, 2010.

LONGUI, E. L.; LIMA, I. L.; FLORSHEIM, S. M. B.; BUFOLO, A. Variação anatômica radial do lenho de açoitacavalo (Luehea divaricata) e sua influência na densidade aparente. Revista do Instituto Florestal, São Paulo, v. 21, n. 2, p. 181-190, 2009.

LORENZI, H. Árvores brasileiras: manual de identificação e cultivo de plantas arbóreas nativas do Brasil. 4. ed. Nova Odessa: Instituto Plantarum, 2002. v. 1.

MAINIERI, C.; CHIMELO, J. P. Ficha de característica das madeiras Brasileiras. São Paulo: IPT-Divisão de Madeira, 1989.

MALAN, F. S. Eucalypts improvement for lumber production. In: SEMINÁRIO INTERNACIONAL E UTILIZAÇÃO DA MADEIRA DE EUCALIPTO PARA SERRARIA, 1995, São Paulo. Anais... Piracicaba: IPEF; IPT; IUFRO; ESALQ, 1995. p. 1-19.

MALAN, F. S. Variation, association and inheritance of juvenile wood properties of Eucalyptus grandis Hill ex Maiden with special reference to the effect of rate of growth. South African Forestry Journal, Pretoria, v. 157, p. 16-23, 1991.

MALAN, F. S.; HOON, M. Effect of initial spacing and thinning on some wood properties of Eucalyptus grandis. South African Forestry Journal, Pretoria, v. 163, p. 13-20, 1992.

POLLI, H. Q.; REIS, G. G.; REIS, M. G. F.; VITAL, B. R.; PEZZOPANE, J. E. M.; FONTAN, I. C. I. Qualidade da madeira em clone de Eucalyptus grandis W. Hill ex Maiden submetido a desrama artificial. Revista Árvore, Viçosa, v. 30, n. 4, p. 557-566, 2006.

Cerne, Lavras, v. 17, n. 4, p. 517-524, out./dez. 2011 
ROCHA, F. T.; FLORSHEIM, S. M. B.; COUTO, H. T. Z. Variação das dimensões dos elementos anatômicos da madeira de árvores de Eucalyptus grandis Hill ex Maiden aos sete anos. Revista do Instituto Florestal, São Paulo, v. 16, n. 1, p. 43-55, 2004.

SAS INSTITUTE. SAS procedures guide. Version 8. Cary, 1999.

SEBBENN, A. M.; SIQUEIRA, A. C. M. F.; GURGELGARRIDO, L. M. A.; ANGERAMI, E. M. R. A. Variabilidade genética e interação genótipo x locais em Jequitibá-rosaCariniana legalis (Mart.) O.Ktze. Revista do Instituto Florestal, São Paulo, v. 12, n. 1, p. 13-23, 2000.

SIQUEIRA, A. C. M. F.; SEBBENN, A. M.; ETTORI, L. C.; NOGUEIRA, J. C. B. Variação genética entre e dentro de populações de Balfourodendron riedelianum (Engler) Engler para conservação ex situ. Revista do Instituto Florestal, São Paulo, v. 12, n. 2, p. 89-103, 2000.
URBINATI, C.; AZEVEDO, A. A.; SILVA, E. A. M.; LISBOA, P. L. Variação estrutural quantitativa no lenho de Terminalia ivorensis A. CHEV, Combretaceae. Acta Botanica Brasilica, São Paulo, v. 17, n. 3, p. 421-437, 2003.

WILKES, J. The influence of rate of growth on the density and heartwood extractives content in eucalypt species. Wood Science and Technology, Canberra, v. 18, n. 2, p. 113-20, 1984.

WILKES, J. Variations of wood anatomy within species of Eucalyptus. IAWA Bulletin, Leiden, n. 9, p. 13-23, 1988.

WRIGHT, J. W. A simplified design for combined provenance and progeny testing. Silvae Genetica, Frankfurt, v. 27, n. 2, p. 68-70, 1978.

YANCHUK, A. D.; MICKO, M. M. Radial variation of wood density and fiber length in trembling aspen. IAWA Bulletin, Leiden, v. 11, n. 2, p. 211-215, 1990.

Cerne, Lavras, v. 17, n. 4, p. 517-524, out./dez. 2011 